\title{
Spinal Epidural Abscess in Patients with Sepsis Who Experienced Significant Improvements after Complete Paralysis: Two Case Reports
}

\author{
Tomofumi Ogoshi*, Motoo Yoshimiya, Hiroshi Ichibakase, Takayoshi Kimura, \\ Masafumi Kameoka, Hayato Yoshioka, Takahiro Ueda, Masato Homma \\ Department of Emergency and Critical Medicine, Tottori University Hospital, Yonago, Japan \\ Email: ${ }^{*}$ tomofumi129@hotmail.com
}

How to cite this paper: Ogoshi, T., Yoshimiya, M., Ichibakase, H., Kimura, T., Kameoka, M., Yoshioka, H., Ueda, T. and Homma, M. (2020) Spinal Epidural Abscess in Patients with Sepsis Who Experienced Significant Improvements after Complete Paralysis: Two Case Reports. Open Journal of Emergency Medicine, 8, 125-133. https://doi.org/10.4236/ojem.2020.84014

Received: November 26, 2020 Accepted: December 25, 2020 Published: December 28, 2020

Copyright $\odot 2020$ by author(s) and Scientific Research Publishing Inc. This work is licensed under the Creative Commons Attribution International License (CC BY 4.0).

http://creativecommons.org/licenses/by/4.0/ (c) (i) Open Access

\begin{abstract}
Spinal epidural abscess (SEA) is a rare condition, and a delay in its diagnosis causes paralysis. In this study, we report two rare cases of delayed diagnosis of SEA whose conditions improved after a state of complete paralysis. The first case was a 71-year-old diabetic man who received a corticosteroid injection for shoulder pain that caused intensified pain. Thereafter, the patient developed paralysis of both legs in stage IV according to Heusner staging. Subsequently, he was diagnosed with multiple abscesses and sepsis. He was in a poor state of health. Therefore, we treated his epidural abscess conservatively. After a month, his muscle strength had improved to Heusner stage III-A, and he was transferred to another hospital. The second case was a 64-year-old diabetic man who received an epidural corticosteroid injection for lower back pain. However, the pain intensified and was admitted to the hospital for pyelonephritis. He developed paralysis in both of his arms and legs presenting as a Heusner stage IV, caused by a cervical epidural abscess. A laminoplasty was performed and paralysis was improved. However, the patient subsequently developed a left subcortical hemorrhage. He underwent surgery. However, his right hemiplegia persisted, and on Day 21, he was transferred back to the previous hospital with a Heusner III-A. We could improve the patients' paralysis by cooperating closely with infectious disease specialists and spine surgeons, taking intensive care, applying antibacterial agents appropriately, and operating quickly.
\end{abstract}

\section{Keywords}

Antibacterial Agents, Diabetes Mellitus, Iatrogenic Abscesses, Infection, Spinal Epidural Abscess 


\section{Introduction}

Spinal epidural abscess (SEA) is rare [1], with a small proportion of patients. Approximately, all with SEA have a classical diagnostic triad of fever, back pain, and neurologic deficits. According to this classical triad, diagnosis of SEA is sometimes delayed [2] [3]. Patients who may become severely paralyzed rarely improve. In this study, we report two patients with multiple abscesses, delayed diagnoses, and sepsis. They got better due to the collaboration between doctors of orthopedics, infectious disease specialists, and emergency medicine. We also considered the reasons for delayed diagnosis and improvement in paralysis in light of the published literature.

\section{Case Presentation}

Case 1: A 71-year-old man with a history of uncontrolled diabetes presented to the Surgery Clinic A with right shoulder pain and no fever. He was administered medications. Since his pain was not alleviated, corticosteroids were injected into his shoulder joint. Three days later, pain spread from his right upper limb to his neck, and he experienced difficulty in speaking. Therefore, he was examined in the Internal Medicine Department at Hospital B. He was found to be hyperglycemic. Although his body temperature had not been taken, and he was referred and admitted to Hospital C. Computed tomography (CT) scan revealed abscesses in his right trapezius and deltoid muscles, and he was referred to the orthopedics department. The abscesses in the deltoid were aspirated with a needle, and he was treated with antibiotics (cefazolin $3 \mathrm{~g} /$ day + minocycline 2 $\mathrm{g} /$ day). Two days later, the abscess near his clavicle increased in size. An incision was made under local anesthesia, and the wound was irrigated through this incision. The antibiotic was switched to meropenem ( $3 \mathrm{~g} /$ day). Although laboratory data indicated severe inflammation, his blood glucose level was controlled. On day 5 of hospitalization, he developed paralysis of both legs in Heusner stage IV. Magnetic resonance imaging (MRI) revealed an epidural abscess (level 4 - 9). Therefore, he was referred to our hospital for surgery.

On admission, the patient could communicate verbally, and his airway was patent. He had a respiratory rate of 25 breaths/min, partial pressure of oxygen $\left(\mathrm{PaO}_{2}\right)$ of $87.8 \mathrm{mmHg}$ ( $3 \mathrm{~L}$ of $\mathrm{O}_{2}$ was administered through a mask), blood pressure of $127 / 60 \mathrm{mmHg}$, heart rate of $114 \mathrm{bpm}$, Glasgow Coma Scale (GCS) score of $\mathrm{E} 4, \mathrm{~V} 4$ and $\mathrm{M} 6$, and a temperature of $39.0^{\circ} \mathrm{C}$. Local findings included swelling and warmth in the right shoulder and a $5-\mathrm{cm}$ wound on the anterior aspect of the clavicle. Although he had no muscle strength in his legs below the level of the iliopsoas muscles and no sensation below T4, muscle strength in his arms was normal. The tendon reflexes were normal in his arms but absent in his legs. His blood test results on admission revealed an elevated white blood cell (WBC) count $(10,100$ cells $/ \mu \mathrm{L})$, elevated C-reactive protein (CRP; $18.4 \mathrm{mg} / \mathrm{dL})$ levels, platelet counts (plt; $\left.122 \times 10^{3} / \mu \mathrm{l}\right)$, and procalcitonin (PCT) levels $(1.01 \mathrm{ng} / \mathrm{ml}$ ). He had mild liver dysfunction: alanine aminotransferase (ALT), $128 \mathrm{U} / \mathrm{L}$; and 
aspartate aminotransferase (AST), $73 \mathrm{U} / \mathrm{L}$ levels. In addition, his serum potassium levels were slightly elevated $(5.2 \mathrm{mg} / \mathrm{dL})$. He had a disseminated intravascular coagulation (DIC) score of 2 points (prothrombin time international normalized ratio [PT-INR], 1.95 and D-dimer level, $12.6 \mu \mathrm{g} / \mathrm{mL}$ ), quick sepsis-related Sequential Organ Failure Assessment (qSOFA) score of 2 points, Sequential Organ Failure Assessment (SOFA) score of 6 points, and an Acute Physiology and Chronic Health Evaluation (APACHE) II score of 27 points. A plain CT scan revealed an abscess near his right shoulder, and abscesses in both the iliopsoas muscles and prostate. Plain MRI revealed changes in brightness of the T6 and T7 vertebrae, and an epidural abscess extending from T4 to T9 (Figure 1). The patient was scheduled to undergo a laminectomy that same day to treat the epidural abscess. However, the surgery was postponed because of his poor clinical condition. The abscess near the shoulder alone was irrigated and debrided. The following day, the abscesses of both the iliopsoas muscles were drained, and the prostatic abscess was treated conservatively. We suggested that he had a methicillin-resistant Staphylococcus aureus (MRSA) infection, and hence, we treated him with vancomycin ( $1 \mathrm{~g} /$ day), whereas the prostatic abscess was treated with clindamycin (1800 mg/day).On day 2, DIC score was 3 point and SOFA score was 11 point because blood pressure was getting down and consciousness was extremely low. On day 3 of hospitalization, his fever persisted. However, his DIC score was 2 points and SOFA score was 6points. The pus was aspirated from his prostatic abscess, and Methicillin-sensitive Staphylococcus aureus (MSSA) was cultured from it. Following this, antibiotic therapy was switched to ceftriaxone (4 g/day) plus clindamycin $(1800 \mathrm{mg} /$ day $)$, and he gradually regained strength in his legs. On day 9 , his fever persisted, and a CT scan revealed pooling of exudate around his right shoulder joint, which was drained and identified as serous exudate. On day 14, the shoulder incision was irrigated and debrided. A month later, his muscle strength had improved to grade 3 on manual muscle testing presenting as Heusner stage III-A. An MRI indicated that the epidural abscess was shrinking, and sepsis improved. Therefore, he was transferred to Hospital C on Day 31 (Figure 2).
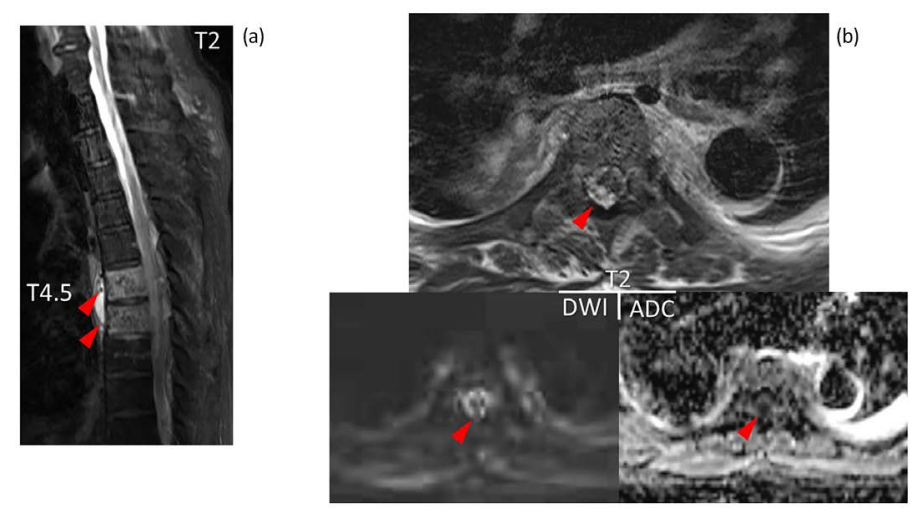

Figure 1. Imaging performed for case 1 at admission. Magnetic resonance imaging (T2) image showing spondylitis at T6 and T7. DWI, diffusion weighted image; ADC, apparent diffusion coefficient. 


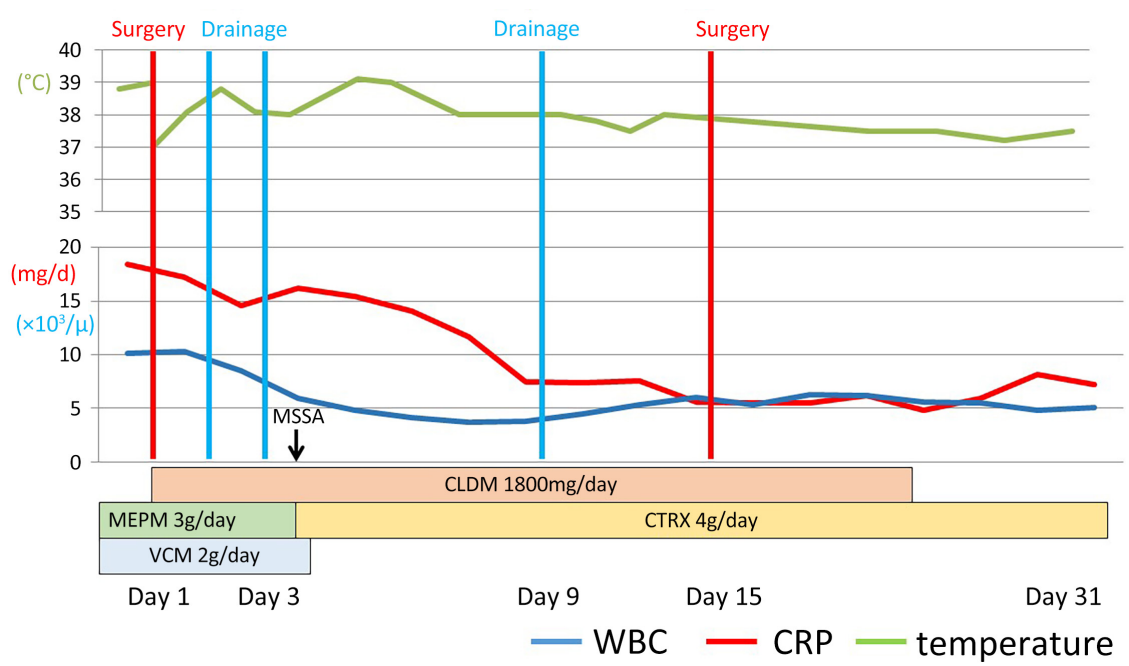

Figure 2. Clinical course of case 1. CLDM, clindamycin; CTRX, ceftriaxone; MEPM, meropenem; VCM, vancomycin.

Case 2: A 64-year-old man with a history of uncontrolled diabetes and hypertension who developed lower back pain with no precipitating cause and no fever was examined at Hospital A. He received an epidural block containing corticosteroids. His lower back pain was temporarily alleviated but intensified 2 days later. He was examined in the orthopedic clinic at Hospital B, where he was administered another epidural block containing corticosteroids. Two days later, he developed pain in his neck and lower back, and was examined in the internal medicine clinic at Hospital B. Severe inflammation was noted, and pyelonephritis was suspected. Therefore, he was admitted to the same hospital. His neck pain further intensified, and he was evaluated by an orthopedic specialist. An abscess was noted near the atlantoaxial vertebrae, which was drained with a needle. Meropenem ( $3 \mathrm{~g} /$ day) was administered to treat the sepsis, and recombinant human soluble thrombomodulin (rTM) $380 \mathrm{U} / \mathrm{kg}$ and antithrombin (1500 U/day) were administered to treat his DIC. He experienced gradual onset of numbness in his fingers and was referred to our hospital for further management. On admission, he could communicate verbally, and his airway was patent. He had a respiratory rate of 22 breaths $/ \mathrm{min}, \mathrm{PaO}_{2}$ of $79.8 \mathrm{mmHg}(2 \mathrm{~L}$ by nasal cannula), blood pressure of $169 / 109 \mathrm{mmHg}$ with administration of a vasopressor, heart rate of $107 \mathrm{bpm}$, GCS scores of E4, V5 and M6, and temperature of $36.6^{\circ} \mathrm{C}$. Local findings were slight pain at the back of the neck on rotation to the right and left. Although he had numbness along the index finger to the ring finger of his right hand, muscle strength in his arms and legs was normal. He also had pain in his right shoulder, which increased on extension. On admission, he had a WBC count of 13,100 cells/ $\mu \mathrm{L}, \mathrm{CRP}$ levels of $31.21 \mathrm{mg} / \mathrm{dL}$, and PCT levels of $\geq 2$ $\times 10^{3} / \mu \mathrm{L}$. He had an acute DIC score of 4 points (Plt, $102 \times 10^{3} / \mu \mathrm{L}$; PT-INR, 1.23 min; and D-dimer, $5.9 \mathrm{ng} / \mathrm{ml}$ ), qSOFA score of 2 points and SOFA score of 9 points and an APACHE II score of 14 points.

A CT scan revealed an abscess at the back of his oropharynx, abscesses in the 
iliopsoas muscles, an abscess in his left piriformis muscle, and thrombosis in both common iliac veins. MRI revealed pooling of fluid around the atlantoaxial joint, around the posterior arch of the atlas, and in the retropharyngeal space along with epidural abscesses at C1-2 and L4-5 (Figure 3, Figure 4). Thus, meropenem ( $3 \mathrm{~g} /$ day) and rTM $(380 \mathrm{U} / \mathrm{kg})$ were administered by the patient's previous physician. After admission, the patient's general condition improved. In the morning of day 2 of hospitalization although hypoesthesia and paralysis were noted in his arms and legs in Heusner stage IV, the DIC score was 4 points and the SOFA score was 6 points. An emergency MRI revealed no marked change in the epidural abscess at C1-2 and L4-5. The area of the spinal canal (C1-2) was still severely inflamed, and his paralysis worsened. Thus, linezolid (1200 mg/day) was administered for possible MRSA infection. We diagnosed the patient with paralysis caused by the C1-2 abscess because abscess of L 4-5 abscess was unclear and did not compress the nerves. Therefore, C1 laminectomy and C2-6 laminoplasty were performed on the same day, 5 hours after paralysis. During surgery, an abscess was noted near the vertebral arches of C1-3. The abscess culture revealed MSSA. On day 4 of hospitalization, his antibiotic therapy was switched to sulbactam/ampicillin (12 g/day). Bilateral empyema developed and was drained on day 5 of hospitalization. The DIC score was 3 points, and the SOFA score was 3 points. Although the patient had a reduced level of consciousness and right hemiplegia on day 11 of hospitalization, his paralysis subsequently improved to a Heusner stage III-A. A head CT scan revealed a subcortical hemorrhage on the left side of the brain, and the patient was diagnosed with a ruptured infectious intracranial aneurysm. The aneurysm in the left middle cerebral artery was then clipped, and a hematoma was removed. On day 17 of hospitalization, a CT scan revealed a residual exudate in the iliopsoases. Thus, CT-guided drainage was performed. Although his right hemiplegia remained in Heusner stage III-A, he was treated conservatively. The inflammation decreased, and the patient could use a wheelchair. On day 23 of hospitalization, he was transferred to Hospital B (Figure 5).
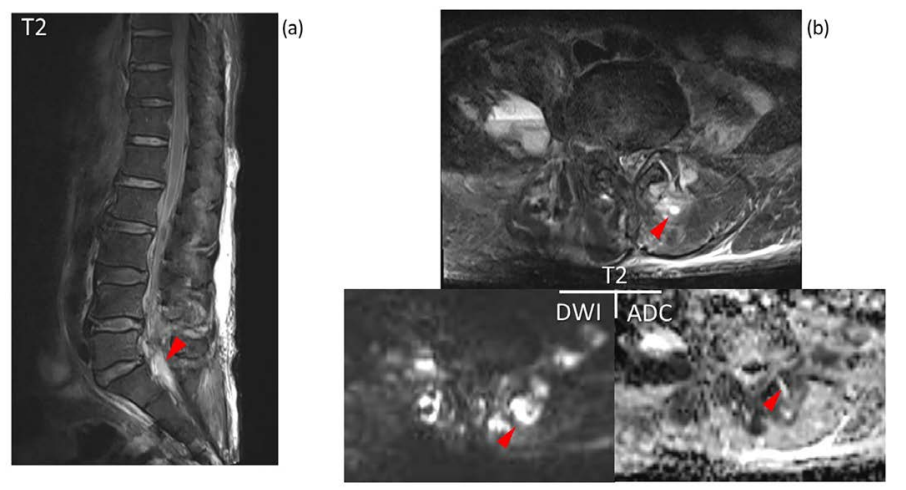

Figure 3. Imaging of the lumbar spine performed for case 2. Magnetic resonance imaging of the lumbar spine showing epidural abscesses and pooling of fluid around the atlantoaxial joint, around the posterior arch of the atlas, and in the retropharyngeal space at admission. 


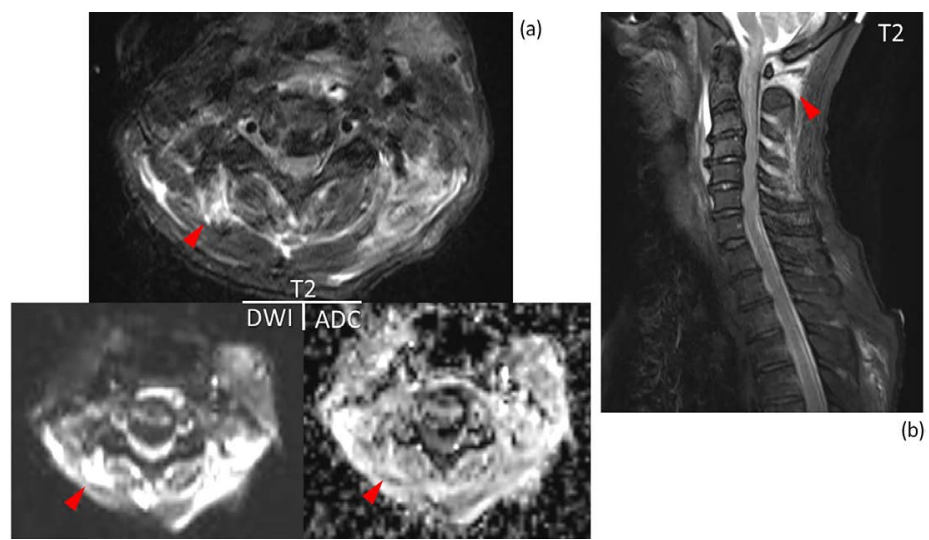

Figure 4. Imaging of the cervical spine performed for case 2. Magnetic resonance imaging of the cervical spine showing pooling of fluid around the atlantoaxial joint, around the posterior arch of the atlas, and in the retropharyngeal space at admission.

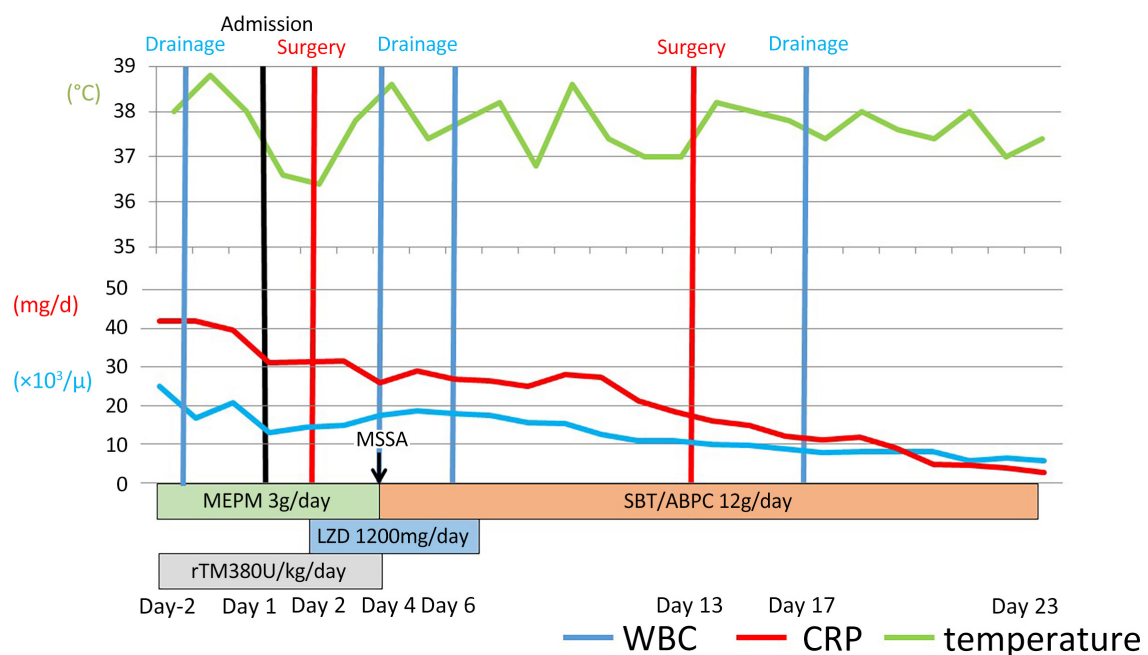

Figure 5. Clinical course of case 2. LZD, linezolid; SBT/ABPC, sulbactam/ampicillin; rTM, recombinant human soluble thrombomodulin.

\section{Discussion}

SEA is rare, with an annual incidence rate of 0.88 cases per 100,000 people. However, it has been noticed in recent years that this incidence of SEA has been increasing [1] [4]. SEA is a severe infection, with a mortality rate of 5\% - 7\% [5] [6], and with paraplegia occurring in $37 \%$ of these patients [7]. The initial symptoms of SEA are usually nonspecific. The classical diagnostic triad is fever, back pain, and neurologic defects. However, only $7.9 \%$ of patients have all three symptoms [2] [7] [8]. Back pain is the most common symptom and is present in $70 \%-100 \%$ of cases. Only $48 \%$ of SEA patients have had fever [8]. In many instances, other medical disorders or other abscesses are noted, or neurological symptoms are also not evident. Therefore, all of these factors contribute to delaying diagnosis of SEA in 75\% of cases [4], leading to delayed or missed diagnosis [7]. The correct diagnosis rate upon the first visit is only $24.2 \%$ [9]. Yuya et 
al. reported that the period from onset of pain to the diagnosis is average 8.6 days [10]. Our diagnosis of Case 1and Case 2 took 16 and 7 days, respectively.

The two patients did not present with fever upon their initial visit, with Case 1 only experiencing neck and lower back pain and Case 2 experiencing lower back pain. Therefore, case 1 was only diagnosed with shoulder infection and case 2 was diagnosed with pyelonephritis, and this delayed the diagnosis of SEA. If patients with skeletal pain present with risk factors: vertebral osteomyelitis, epidural catheter, paraspinal injections of glucocorticoid or analgesics, alcoholism, diabetes, or HIV infection, SEA should be considered [6] [11] [12] [13].

There were three reasons for the delayed diagnosis of SEA in these cases: 1) there was no classical diagnostic triad comprising fever, back pain, and neurologic deficits, with fever being especially absent; 2 ) there were multiple abscesses, but it was considered that only one abscess caused sepsis in each patient; 3 ) and there was incomplete medical history, particularly with respect to diabetes and treatments.

Both patients were elderly men with poorly controlled diabetes, which made them susceptible to infection. In addition, diabetes status had not been assessed in both cases, and they had previously received corticosteroid injections for pain. One case had received a corticoid injection at the shoulder joint, while the other had received an epidural corticoid injection for a short duration, with both patients experiencing sepsis after injection.

A previous study conducted by Gregori et al. has indicated that it was important to take a methodological approach toward deciding on an accurate and prompt diagnosis: a multidisciplinary team of physicians, including infectious disease specialists, neuroradiologists and spine surgeons is necessary to treat SEA [14]. This is because a delay in diagnosis for more than 24 hours from the onset of paralysis has been shown to increase the risk of paralysis compared to cases diagnosed immediately [2]. Many patients (80\%) who underwent surgery after 24 hours experienced bladder-rectal disorders, while only $10 \%$ of patients who underwent surgery within 24 hours had complications. Therefore, early diagnosis is important. If complete paralysis (Heusner stage IV) occurs, only $14 \%$ of patients' conditions improved [15]. A study by Kenji et al. [16] reported that patients presenting with a stage below Heusner stage III did not improve postoperatively. A study by Katsuki et al. [15] reported A multidisciplinary approach involving both a spinal surgeon and an infectious disease specialist was necessary to better define the treatment strategy for the spontaneous spinal infection. The patient in case 1 was paralyzed and in poor clinical condition, a poor surgical candidate. Case 2 was initially at Heusner stage 1. Therefore, antibiotics were administered and he was managed in intensive care and got better. However, after a few days, he suddenly got worse and had paralysis. An operation was performed 5 hours after paralysis. The patient was able to improve to a Heusner stage III-A. We were in constant communication with infectious disease specialists and spine surgeons to treat patients quickly and appropriately. 


\section{Conclusion}

In conclusion, we report rare two rare cases of diabetes whose diagnoses of SEA were delayed. However, their paralysis improved to a Heusner IV. To treat SEA quickly and appropriately, it is vital to cooperate with infectious disease specialists and spine surgeons.

\section{Acknowledgements}

We would like to thank Masaki Yoshida, Chikako Takeda, Tokumitsu Mihara, Kensaku Okada, Tsuyoshi Kitaura, Masaki Nakamoto, Shinji Tanishima, Hideki Nagashima and Hiroki Chikumi for treating the patients featured in this article.

\section{Consent for Publication}

The patients provided written informed consent.

\section{Conflicts of Interest}

The authors declare no conflicts of interest regarding the publication of this paper.

\section{References}

[1] Ptaszynski, A.E., Hooten, W.M. and Huntoon, M.A. (2007) The Incidence of Spontaneous Epidural Abscess in Olmsted County from 1990 through 2000: A Rare Cause of Spinal Pain. Pain Medicine, 8, 338-343. https://doi.org/10.1111/j.1526-4637.2006.00173.x

[2] Davis, D.P., Wold, R.M., Patel, R.J., Tran, A.J., Tokhi, R.N., Chan, T.C., et al. (2003) The Clinical Presentation and Impact of Diagnostic Delays on Emergency Department Patients with Spinal Epidural Abscess. Journal of Emergency Medicine, 26, 285-291. https://doi.org/10.1016/j.jemermed.2003.11.013

[3] Bond A. and Manian F. (2016) Spinal Epidural Abscess: A Review with Special Emphasis on Earlier Diagnosis. Biomed Research International, 2016, Article ID: 1614328. https://doi.org/10.1155/2016/1614328

[4] Zarghooni, K., Röllinghoff, M., Sobottke, R. and Eysel, P. (2012) Treatment of Spondylodiscitis. International Orthopaedics, 36, 405-411. https://doi.org/10.1007/s00264-011-1425-1

[5] Vakili, M. and Crum-Cianflone, N.F. (2017) Spinal Epidural Abscess: A Series of 101 Cases. American Journal of Medicine, 130, 1458-1463. https://doi.org/10.1016/j.amjmed.2017.07.017

[6] Darouiche, R.O. (2006) Spinal Epidural Abscess. The New England Journal of Medicine, 355, 2012-2020. https://doi.org/10.1056/NEJMra055111

[7] Chen, W.C., Wang, J.L., Wang, J.T., Chen, Y.C. and Chang, S.C. (2008) Spinal Epidural Abscess due to Staphylococcus aureus. Clinical Manifestations and Outcomes. Journal of Microbiology, Immunology and Infection, 41, 215-221.

[8] Curry, W.T., Hoh Jr., B.L., Amin-Hanjani, S. and Eskandar, E.N. (2005) Spinal Epidural Abscess: Clinical Presentation, Management, and Outcome. Surgical Neurology, 63, 364-371. https://doi.org/10.1016/j.surneu.2004.08.081

[9] Hlavin, M.L., Kaminski, H.J., Rose, J.S. and Ganz, E. (1990) Spinal Epidural Ab- 
scess: A Ten Year Perspective. Neurosurgery, 27, 177-184.

https://doi.org/10.1227/00006123-199008000-00001

[10] Yuya, E., Hiroki, T., Rei, S., Shunichi, M., Yumi, T., Morika, S., et al. (2017) Spinal Epidural Abscess with Rapidly Progressing Paralysis. Sendai Medical Center Journal, 7, 57-67.

https://nsmc.hosp.go.jp/Journal/2017-7/SMCJ2017-7_casereport02.pdf

[11] Cook, T.M., Counsell, D. and Wildsmith, J.A. (2009). Major Complications of Central Neuraxial Block: Report on the Third National Audit Project of the Royal College of Anesthetists. British Journal of Anaesthesia, 102, 179-190.

https://doi.org/10.1093/bja/aen360

[12] Gaul, C., Neundörfer, B. and Winterholler, M. (2005) Iatrogenic (Para-)Spinal Abscesses and Meningitis Following Injection Therapy for Low Back Pain. Pain, 116, 407-410. https://doi.org/10.1016/j.pain.2005.05.032

[13] Krishnamohan, P. and Berger, J.R. (2014) Spinal Epidural Abscess. Current Infectious Disease Reports, 16, Article No. 436. https://doi.org/10.1007/s11908-014-0436-7

[14] Gregori, F., Grasso, G., Iaiani, G., Marotta, N., Torregrossa, F. and Landi, A. (2019) Treatment Algorithm for Spontaneous Spinal Infections: A Review of the Literature. Craniovertebral Junction and Spine, 10, 3-9. https://doi.org/10.4103/jcvjs.JCVJS_115_18

[15] Katsuki, S., Masao, K. and Kouhei, M. (2002) A Case of Epidural Abscess Early after Rectal Cancer Surgery. Journal of Otolaryngology of Japan, 105, 1143-1146. https://doi.org/10.3950/jibiinkoka.105.1143

[16] Kenji, T., Masahiko, I. and Yoshihiro, T. (1996) The Experience of Treatment for Spinal Epidural Abscess. Central Japan Journal of Orthopaedic Surgery and Traumatology, 39, 1055-1056. 